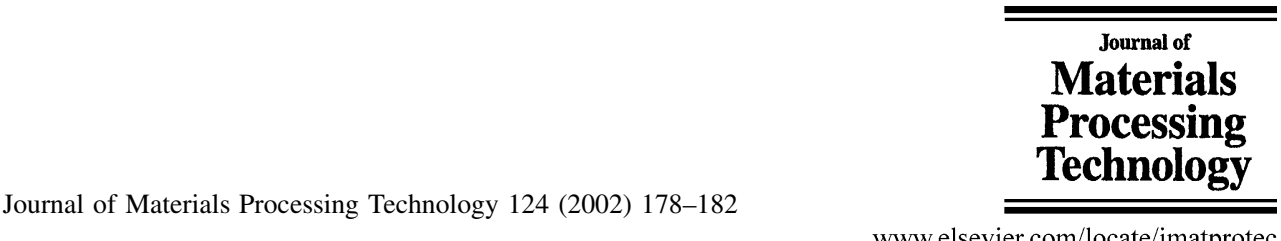

www.elsevier.com/locate/jmatprotec

\title{
Analysis of asymmetrical cold rolling with varying coefficients of friction
}

\author{
H. Gao ${ }^{a}$, , S.C. Ramalingam ${ }^{a}$, G.C. Barber ${ }^{\mathrm{a}}$, G. Chen ${ }^{\mathrm{b}}$ \\ a Department of Mechanical Engineering, Oakland University, Rochester, MI 48309, USA \\ ${ }^{\mathrm{b}}$ Institute of Mechanics, Chinese Academy of Sciences, Beijing 100080, China
}

Received 28 September 2000; accepted 5 March 2002

\begin{abstract}
In this research, asymmetrical cold rolling was produced by the difference in the coefficient of friction between rolls and sheets rather than the difference of roll radius or rotation speeds. The influence of friction coefficient ratio on the cross shear deformation, rolling pressure and torque was investigated using slab analysis. The results showed that the shear deformation zone length increased with the increase of the friction coefficient ratio. The rolling force decreased only under the condition that the friction coefficient ratio increased while the sum of the friction coefficients was held constant. As the reduction per pass was increased, the shear deformation zone length increased and the rolling force also increased. An increase of the front tension resulted in a decrease of the shear deformation zone length. An increase of back tension, however, led to an increase of the shear deformation zone length. The reduction of rolling torque for the work roll with higher surface roughness was greater than that for the work roll with lower surface roughness. (C) 2002 Elsevier Science B.V. All rights reserved.
\end{abstract}

Keywords: Asymmetrical cold rolling; Frictional coefficient ratio; Slab method

\section{Introduction}

Asymmetrical cold rolling is a process which utilizes different peripheral velocities between two work rolls [1]. In comparison, symmetrical rolling is a process where the peripheral velocity and the radius of the upper roll are equal to those of the lower roll. The velocity difference is due to the variance of diameters or different rotating speeds between the upper and the lower work rolls. The neutral points, where the velocity of the sheet is equal to that of the working roll, become asymmetrical due to the difference of the peripheral velocities of the upper and lower rolls. A third cross shear deformation zone is formed between the backward-slip zone and the forward-slip zone. Both compressive and shear stresses occur simultaneously in the cross shear deformation zone. The advantages of asymmetrical rolling over symmetrical rolling are decreased rolling pressure, reduction of rolling torque and improvement of plate shape. The problems, however, are mill vibration and wrinkles on the plate surface [2].

There exist three different asymmetrical rolling processes. One is produced by using different peripheral velocities with the same roll radius. A second uses different radii with the same angular velocity. The third uses varying coefficients of friction for the two work rolls and keeps the peripheral velocity and roll radius the same for the two work rolls. The

* Corresponding author. first two methods were extensively investigated using the slab method [3,4], upper-bound method [5,6], finite element method [7], stream function method [8] and experimental method [6,9].

For the third asymmetrical rolling method, Johnson and Needham [9] found the effect of the surface roughness of working rolls on the curvature direction of the strip through experimentation. They discovered that for two work rolls with the same radius, when the lower roll had higher surface roughness than the upper roll, the resulting strip was curved downward using a smaller reduction ratio, and upward with a greater reduction ratio. Pospiech [10] also studied the influence of the reduction ratio on the curved direction when the friction conditions were asymmetrical. His initial research on the effect of friction was primarily focused on experiments and curvature shapes. For the situations with varying rolling velocity or different roll radii, the coefficients of friction between the upper roll and the sheet as well as that between the lower roll and the sheet must be different. The previous investigations assumed that the upper frictional coefficient was equal to the lower one. The influence of the frictional coefficient ratio on asymmetrical rolling has not been studied in detail.

The objective of the present research is to study the characteristics of asymmetrical cold rolling produced by different coefficients of friction using the slab method and to gain insight into the main factors which influence asymmetrical cold rolling. 


\section{Mathematical models}

\subsection{Rolling pressure}

\subsubsection{Governing equation}

Before presenting the mathematical model, first consider the assumptions which were made for the asymmetric slab method analysis: The rolls are rigid, the sheet is elasticplastic. The plastic deformation occurs under plane strain conditions (no width expansion). The stresses are uniform in the contact area, the rolling pressure and the horizontal stress produced by the front and back-tension force are considered to be principal stresses. The friction coefficients between the rolls and the sheets are constant along the contact length. The friction coefficient between the upper roll and the sheet can be different from that between the lower roll and the sheet. The friction force in the contact area follows Coulomb's law. The plastic flow at the entrance and the exit are assumed to be horizontal. The contact length is small as compared with the roll circumference. Fig. 1 shows the schematic diagram of asymmetrical cold rolling. There are three zones between the two rolls. I is the backward-slip zone, II is the shear deformation zone, III is the forward-slip zone, $q_{0}$ is the front-tension force, $q_{i}$ is the back-tension force, $h_{i}$ is the original height at the entrance position, and $h_{0}$ is the final height at the exit position. Fig. 2 illustrates the stress distribution of the slab element. $p$ and $\tau$ are normal and tangential stresses on the contacting surfaces respectively. According to the balance of forces along $x$ and $y$ directions respectively,

$\sum X=0, \quad \frac{\mathrm{d}(h q)}{\mathrm{d} x}+2 p \tan \theta-\left(\tau_{1}+\tau_{2}\right)=0$

$\sum Y=0, \quad p=p_{1}\left(1+\mu_{1} \tan \theta\right)=p_{2}\left(1+\mu_{2} \tan \theta\right)$

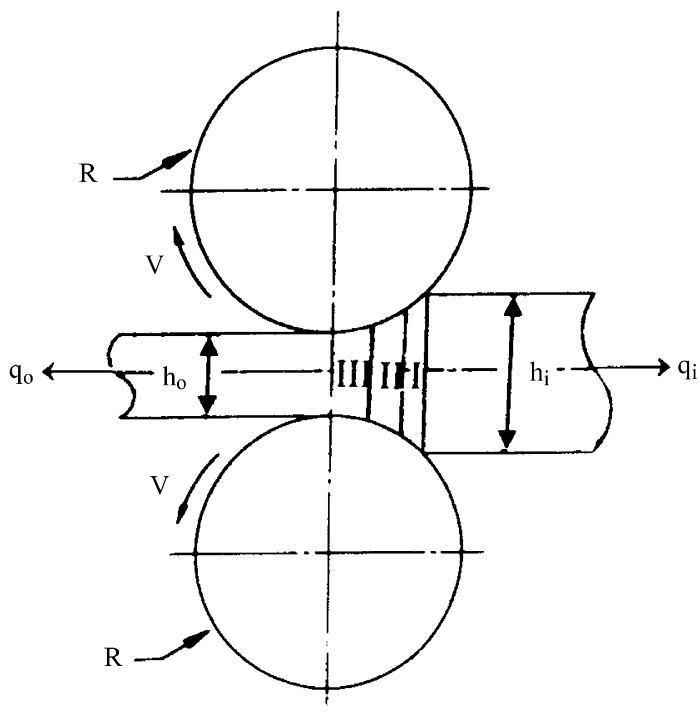

Fig. 1. Schematic diagram of asymmetrical cold rolling caused by different frictional coefficients.

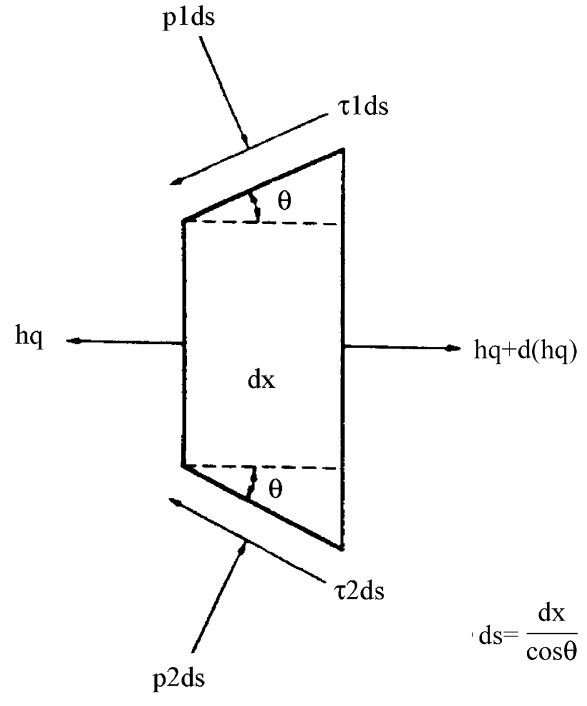

Fig. 2. Slab element in region II.

Combining (1) and (2) with the von Mises yielding criterion

$p+q=2 k$

and the geometry conditions

$\frac{\mathrm{d} h}{\mathrm{~d} x}=\frac{x}{R}$

$h=h_{0}+\frac{x^{2}}{R}$

we obtain the rolling pressure equation

$\bar{p}=C \mathrm{e}^{-\bar{\mu} \bar{x}}+\left(\frac{\bar{x}^{3}}{3 \bar{\mu}}-\frac{\bar{x}^{2}}{\bar{\mu}^{2}}+\left(\frac{1}{\bar{\mu}}+\frac{1}{\bar{\mu}^{3}}\right) \bar{x}-\left(\frac{1}{\bar{\mu}^{2}}+\frac{1}{\bar{\mu}^{4}}\right)\right)$

where

$\bar{p}=\frac{p}{2 k}, \quad \bar{\mu}=\mu_{\mathrm{e}} \sqrt{\frac{R}{h_{0}}} \quad \mu_{\mathrm{e}}=\mu_{1}+\mu_{2}$,

$\bar{x}=\arctan \frac{x}{\sqrt{R h_{0}}}$

and $C$ is a constant, $\mu_{\mathrm{e}}$ is the equivalent coefficient of friction, $R$ is the roll diameter, and $x$ is the distance from the vertical line through the centers of the upper and the lower rolls.

\subsubsection{Boundary conditions}

For the forward-slip zone,

$0 \leq x \leq x_{n 2}, \quad \mu_{\mathrm{e}}=\mu_{1}+\mu_{2}$,

$\bar{p}_{0}=1-\frac{q_{0}}{2 k} \quad($ at $x=0)$

For the backward-slip zone,

$x_{n 1} \leq x \leq L, \quad \mu_{\mathrm{e}}=-\mu_{1}-\mu_{2}$,

$\bar{p}_{0}=1-\frac{q_{i}}{2 k} \quad($ at $x=L)$ 
For the shear deformation zone,

$x_{n 2} \leq x \leq x_{n 1}, \quad \mu_{\mathrm{e}}=\mu_{1}-\mu_{2} \quad\left(\mu_{1}>\mu_{2}\right)$,

$\bar{p}_{\mathrm{s}}=\bar{p}_{\mathrm{f}} \quad\left(\right.$ at $\left.x=x_{n 2}\right), \quad \bar{p}_{\mathrm{s}}=\bar{p}_{\mathrm{b}} \quad\left(\right.$ at $\left.x=x_{n 1}\right)$

From the boundary conditions, we can solve for the constant C.

\subsection{Rolling force}

The rolling force is the summation of forces in three deformation zones:

$P=P_{\mathrm{b}}+P_{\mathrm{s}}+P_{\mathrm{f}}$

$P_{i}=2 k \int_{x 1}^{x 2} f \mathrm{~d} x$

where $P_{i}$ is the rolling force in each deformation zone and $k$ is the shear strength of the sheet metal.

\subsection{Rolling torque}

The rolling torque is an important parameter which determines the energy consumption of the rolling mill:

$T_{1}=\mu_{1} R_{1}\left(P_{\mathrm{b}}-P_{\mathrm{s}}-P_{\mathrm{f}}\right)$

$T_{2}=\mu_{2} R_{2}\left(P_{\mathrm{b}}+P_{\mathrm{s}}-P_{\mathrm{f}}\right)$

where $T_{1}$ and $T_{2}$ are the rolling torques of the higher frictional coefficient roll and the lower frictional coefficient roll, respectively.

\section{Results and discussion}

From the mathematical models, the influencing factors on the asymmetrical rolling were calculated for $R=100 \mathrm{~mm}$, $h_{0}=4.5 \mathrm{~mm}$ and $2 k=50.0 \mathrm{kgf} / \mathrm{mm}^{2}$. Fig. 3 shows the

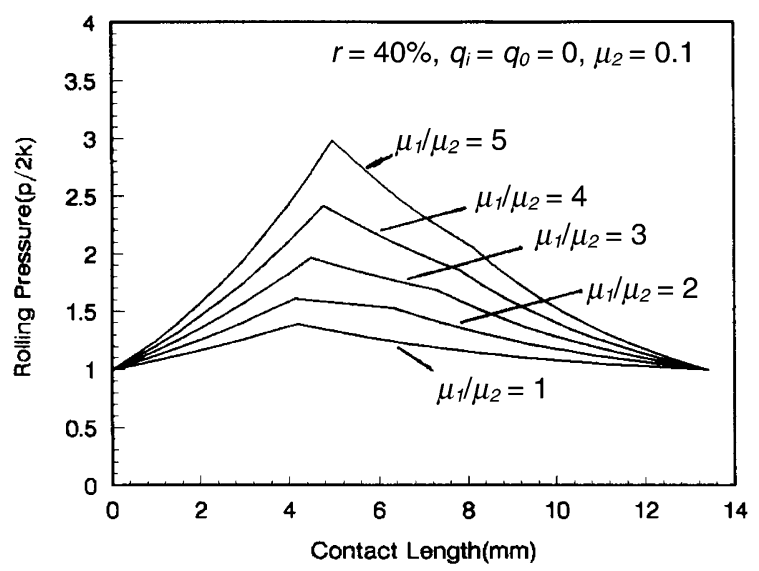

Fig. 3. The effect of friction coefficient ratio on cross shear deformation zone length and rolling pressure.

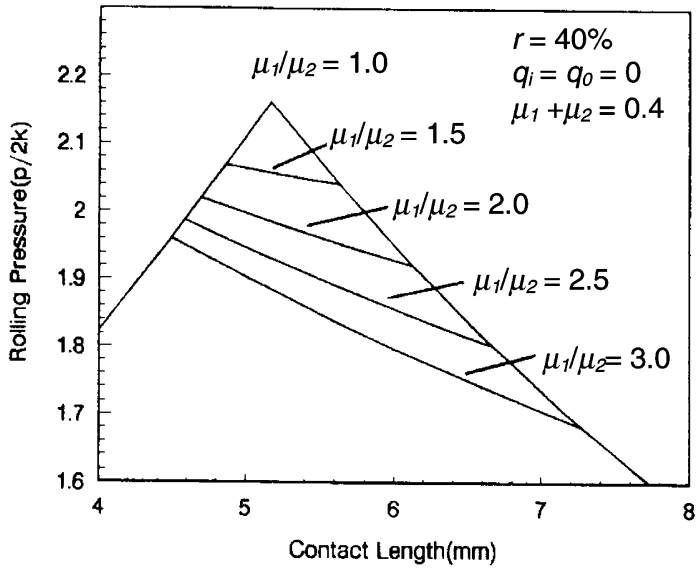

Fig. 4. The influence of friction coefficient ratio on cross shear deformation zone length and rolling pressure with constant sum of frictional coefficients.

effect of the friction coefficient ratio, $\mu_{1} / \mu_{2}$, on the shear deformation and the rolling pressure under the condition that $\mu_{2}$ remains constant. $\mu_{1}$ is varied to change the friction coefficient ratio. $r$ is the rolling reduction per pass. It is seen that the shear deformation zone is enlarged with the increase of the friction coefficient ratio. Furthermore, the rolling pressure also increases when this ratio increases. Now consider the case when the total value of the friction coefficients is kept constant. Fig. 4 shows the changes of the rolling pressure with the friction coefficient ratio. It can be seen that the shear deformation zone becomes longer with an increase in the friction coefficient ratio. An important result is that the rolling pressure decreases with increasing friction coefficient ratio, contrary to the condition shown in Fig. 3. Although the "pressure hills" disappear due to the asymmetrical rolling, it is not always true that the rolling force reduces with the increase of the friction coefficient ratio. The most important factor is the sum of the friction coefficients, not the friction coefficient ratio.

Fig. 5 shows the influence of the tension force on the rolling pressure for asymmetrical rolling. When the tension force is applied, the rolling pressure is obviously reduced. At the same time, with the increase of the front-tension force the shear deformation zone becomes shorter and eventually disappears, as shown in Fig. 5(a). With increasing backtension force, on the other hand, the shear deformation zone becomes longer and the rolling force is reduced further. Therefore, the effect of the back-tension force on asymmetrical rolling is more appreciable than that of the fronttension force.

Fig. 6 shows the effect of the reduction per pass on the rolling pressure and the shear deformation zone length. The rolling pressure rises as the increase of the reduction per pass and the shear deformation increases as well. The ratio of the shear deformation to the entire plastic deformation, $\Delta x / L$, becomes larger with an increase in the reduction per pass. During rolling, the reduction per pass should be increased to 

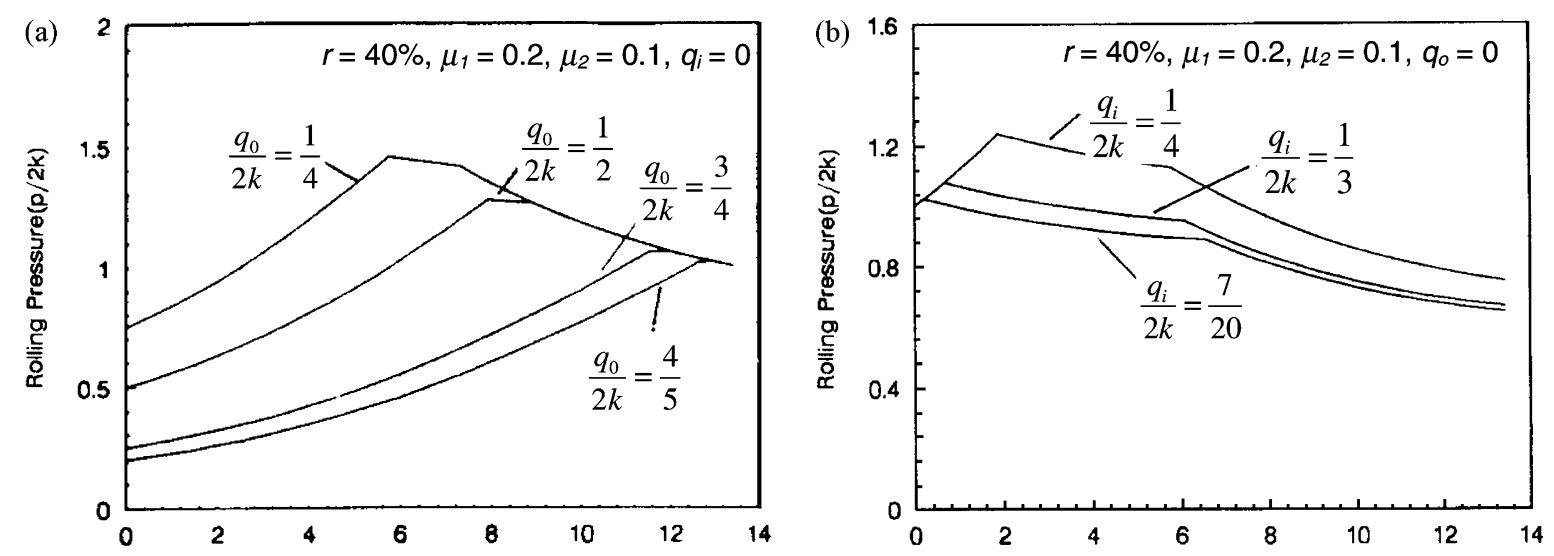

Fig. 5. The influence of tension on rolling pressure and cross shear deformation zone length: (a) front tension; (b) back tension.

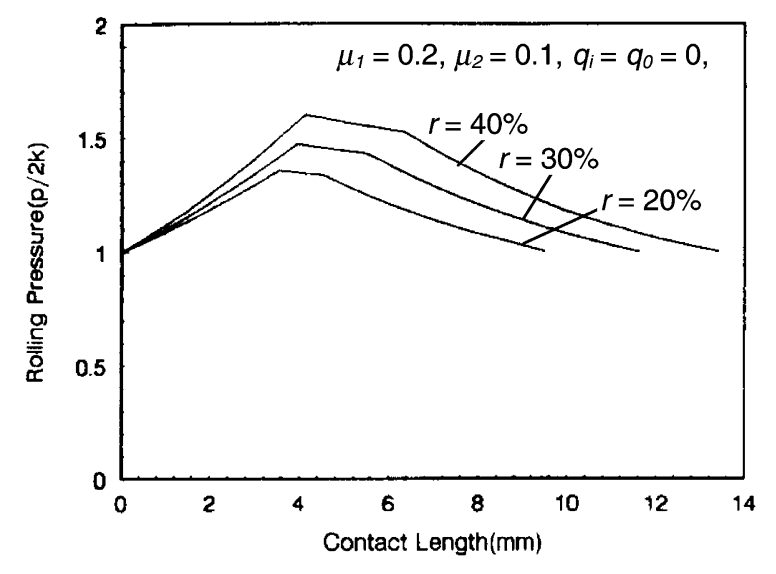

Fig. 6. Variation of rolling pressure and cross shear deformation zone length with reduction under asymmetrical friction condition.

be as large as possible. Rolling very thin steel sheets is not limited by the rolling force but by the rolling pressure. In this situation, asymmetrical rolling plays an important role due to the reduction of the rolling pressure.

Fig. 7 shows the rolling force versus the friction coefficient ratio. As can be seen, the rolling force slightly decreases as the friction coefficient ratio increases. The rolling force is more

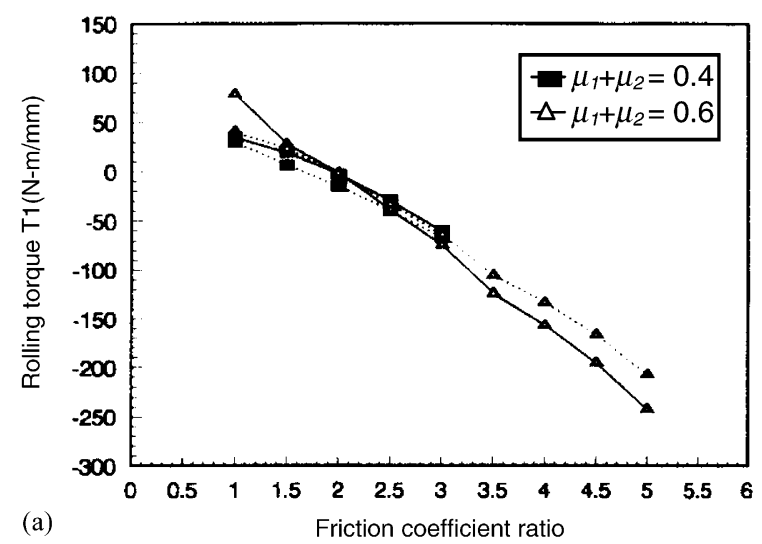

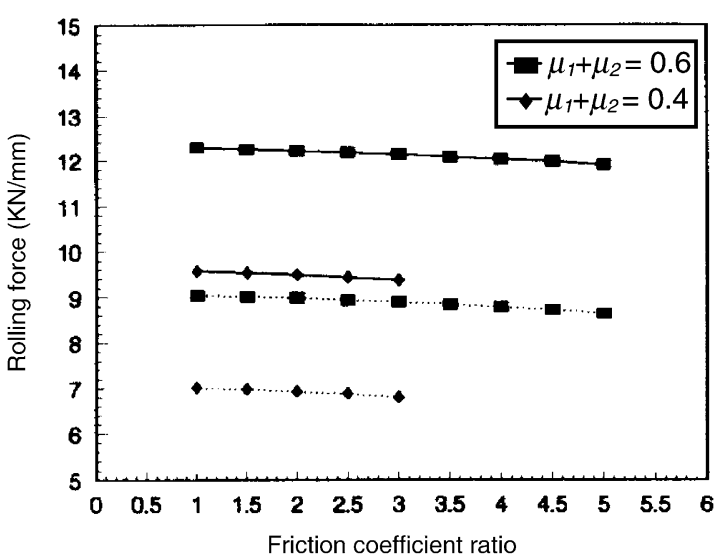

Fig. 7. Variation of rolling force with frictional coefficients.

strongly affected by the sum of the friction coefficients of friction rather than the friction coefficient ratio. The friction coefficient ratio has more influence on the shear deformation than on the rolling force.

Another important influencing factor in rolling is the rolling torque. Fig. 8 shows the influence of the friction coefficient ratio on the rolling torque. As the friction coefficient

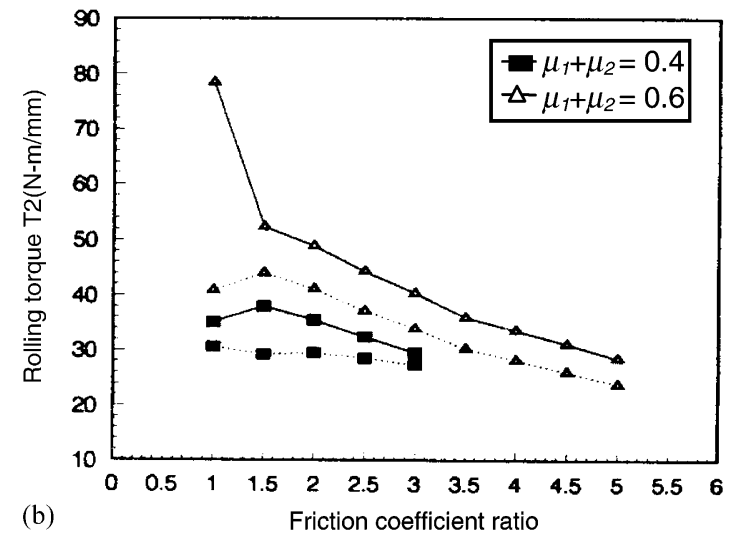

Fig. 8. Variation of rolling torque with frictional coefficient ratio: (a) roll with higher coefficient of friction; (b) roll with lower coefficient of friction. 


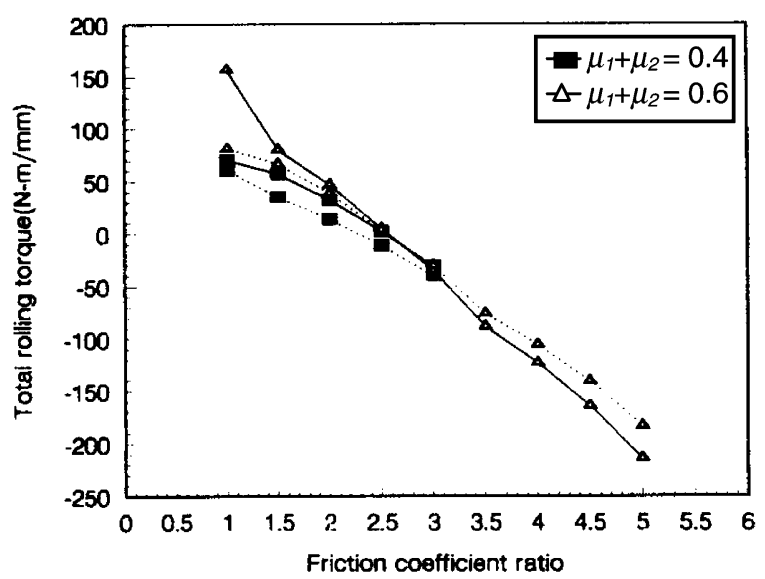

Fig. 9. Variation of total rolling torque with frictional coefficient ratio.

ratio increases, negative rolling torque occurs for the higher roughness work roll. That is, the friction force acts to enhance not to resist the rolling. For the lower roughness work roll, the rolling torque reduces slightly and keeps positive. Accordingly, the total rolling torque reduces due to the effect of asymmetrical rolling, as seen in Fig. 9.

\section{Conclusions}

Using different coefficients of friction, asymmetrical rolling was investigated by applying the slab method. It was found that the shear deformation zone length increased with the increase of the friction coefficient ratio. The rolling force decreased only when the friction coefficient ratio increased while the sum of the friction coefficients was held constant. As the reduction per pass was increased, the shear deformation zone length increased and the rolling force also increased. An increase of the front tension resulted in a decrease of the shear deformation zone length. An increase of back tension, however, led to an increase of the shear deformation zone length. The total rolling torque decreased with the friction coefficient ratio. The decrease of the rolling torque for the work roll with higher surface roughness was greater than that for the work roll with lower surface roughness. With a decrease of the thickness of the sheet and an increase of the coefficient of friction, sticking will occur. Further study is required on the sticking condition with the change of the coefficient of friction.

\section{References}

[1] H. Gao, G. Chen, Asymmetrical cold rolling realized on plain mill for steel sheet by laser-textured rolls, Iron and Steel 33 (3) (1998) 63 66.

[2] A.A. Jafari, A.K. Tieu, A. Basu, Asymmetrical rolling and selfexcited vibrations in a hot roughing mill, Vibrations of Rotating Systems, DE-Vol. 6, ASME, New York, 1993, pp. 193-202.

[3] Y.M. Hwang, G.Y. Tzou, An analytical approach to asymmetrical hot-sheet rolling considering the effects of the shear stress and internal moment at the roll gap, J. Mater. Process. Technol. 52 (1995) 399-424.

[4] G.Y. Tzou, Relationship between frictional coefficient and frictional factor in asymmetrical sheet rolling, J. Mater. Process. Technol. 86 (1999) 271-277.

[5] M. Kiuchi, S.H. Hsiang, The general analytical model in asymmetrical rolling process, in: Proceedings of the 34th Joint Conference of the Japanese Society for the Technology of Plasticity, 1983, pp. 105108.

[6] D. Pan, D.H. Sansome, An experimental study of the effect of rollspeed mismatch on the rolling load during the cold rolling of the strip, J. Mech. Work. Tech. 6 (1982) 361-377.

[7] Y.M. Hwang, T.H. Chen, H.H. Hsu, Analysis of asymmetrical clad sheet rolling by stream function method, Int. J. Mech. Sci. 38 (4) (1996) 443-460.

[8] Z.C. Lin, C.C. Shen, Three-dimensional asymmetrical rolling of an aluminum flat strip using an elastic-plastic finite element method, Int. J. Computer Appl. Technol. 9 (5/6) (1996) 281-297.

[9] W. Johnson, G. Needham, Further experiments in asymmetrical rolling, Int. J. Mech. Sci. 8 (1966) 443-455.

[10] J. Pospiech, A note on the influence of some factors affecting curvature on the flat rolling of strip, J. Mech. Work. Tech. 15 (1987) 69-80. 